\title{
Effect of the Small Punch Test sample geometry on the Liquid Metal Embrittlement of Cu-30 wt\% Zn by the eGaln
}

\author{
Marco Ezequiel ${ }^{1 *}$ and Ingrid Proriol Serre ${ }^{1}$ \\ ${ }^{1}$ Univ. Lille, CNRS, INRAE, Centrale Lille, UMR 8207 - UMET - Unité Matériaux et \\ Transformations, F-59000 Lille, France
}

\begin{abstract}
Liquid metal embrittlement (LME) is the embrittlement or the modification of the fracture behaviour of a metal or alloy when it undergoes plastic deformation while in contact with a liquid metal or liquid alloy. LME occurrence depends strongly on the properties of the metals involved and on the conditions of the mechanical stresses applied to the solid. The Small Punch Test (SPT) on flat specimens is very sensitive to identify the conditions of LME occurrence. Moreover, there are alternative SPT notched specimen geometries that have the potential to screen solid/liquid couples for sensibility to LME in different conditions. To study the apparition of the LME on an alpha brass with $30 \mathrm{wt} \% \mathrm{Zn}$ in contact with the eGaIn (Ga-In eutectic), SPT at room temperature were carried out at different displacement rates and using three specimen types: the standard flat geometry and two notched geometries. While the flat specimens did not present LME, the presence of a notch and a high strain rate induced LME on the other specimen geometries. For these last specimens, the eGaIn modifies the SPT load-displacement curves at the crack propagation stage and changes the fracture to a partially ductile fracture followed by a brittle fracture.
\end{abstract}

\section{Introduction}

A solid metal or alloy that undergoes plastic deformation while in contact with a liquid metal or a liquid alloy may present liquid metal embrittlement (LME). This phenomenon induces a change in the fracture behaviour of the solid and a decrease in its mechanical properties [13]. However, the presence of LME depends on the metals or metallic alloys in contact, specifically on their physicochemical properties, their chemical compositions, and the metallurgical state of the solid. It also depends strongly on external conditions such as the temperature, the strain rate, and the mechanical stress conditions (monotonic, cyclic, bi-axial strain, etc.) $[2,4,5]$.

\footnotetext{
${ }^{*}$ Corresponding author: marco-alejandro.ezequiel-alvarado@univ-lille.fr
} 
Diverse mechanical tests are used to study the LME on solid/liquid couples; one of them is the Small Punch Test (SPT). This miniaturised test allows the assessment of the mechanical behaviour of diverse metals under a wide range of conditions using little material and it is very sensitive to LME [6].

In addition, there are alternative notched specimen geometries designed by [7] and [8] to measure the fracture toughness through the SPT in non-corrosive environments. These alternative notched geometries, not yet used for LME sensitivity studies, could be more suitable to study the sensibility of a solid/liquid couple to LME than the standard flat geometries. Indeed, the introduction of a notch in solid samples increases the sensibility of tensile tests to $\operatorname{LME}[5,9,10]$. For example, on the couples $\mathrm{Cu}-30 \mathrm{wt} \% \mathrm{Zn} / \mathrm{Ga}[5]$ and $\mathrm{T} 91 / \mathrm{Pb}$ $[9,10]$ LME was not present on unnotched tensile specimens; however, on notched tensile specimens, there was a clear apparition of LME.

To study the effect of the alternative SPT notched specimens on the apparition of the LME of a specific solid/liquid couple, we tested the alpha brass $\mathrm{Cu}-30 \mathrm{wt} \% \mathrm{Zn}$ in contact with the Ga-In eutectic (eGaIn) and, for comparison, in air. The alpha brasses are used widely due to their mechanic, electric and thermic properties [11]; and the eGaIn is an alloy that is liquid at room temperature, has good fluidity, has low toxicity, and presents a unique combination of thermic and electric properties $[12,13]$. The LME has been studied on similar couples $(\mathrm{Cu}-30 \mathrm{wt} \% \mathrm{Zn} / \mathrm{Hg}$, beta brass/eGaIn, Cu-30 wt\% $\mathrm{Zn} / \mathrm{Ga})[4,5,14]$, but to our knowledge not in $\mathrm{Cu}-30 \mathrm{wt} \% \mathrm{Zn} / \mathrm{eGaIn}$. Moreover, none of these studies makes use of miniaturised tests such as the SPT, and to date, there are no studies on the use of notched SPT samples to assess LME for any material.

We performed the SPT at different conditions: three specimen geometries (one standard and two notched geometries) and four strain rates. LME sensitivity was studied from the SPT load-displacement curves and the observation of the fractured surfaces after the test.

\section{Materials and methods}

The alpha brass with $30 \mathrm{wt} \% \mathrm{Zn}$ (commercially known as C26000 or cartridge brass) was used in sheet form with an initial thickness of $640 \mu \mathrm{m}$. Its grain size is $18 \pm 3 \mu \mathrm{m}$ and its hardness is $130 \pm 4 \mathrm{HV}_{0,2}$. The eGaIn was elaborated using 99,99\% purity $\mathrm{Ga}$ and $\mathrm{In}$. The alloy was prepared by mixing the eutectic composition $(25,5 \mathrm{wt} \% \mathrm{In})$ by melting $\mathrm{Ga}$ and adding solid In.

The SPT consists in using a 100C6 steel ball to punch the specimens until fracture. For the tests in presence of liquid metal, the upper surface of the specimen, which is submitted to tensile loading, is in contact with the liquid eGaIn. To test the brass sheet with the SPT, three different specimen geometries (Figure 1) were used: the standard SPT geometry with no notch [15], one with a blind longitudinal notch [8], and one with a lateral notch [7]. The thickness of the samples was reduced to $500 \pm 20 \mu \mathrm{m}$ through automatic polishing to fit the European standard on SPT [15] and to avoid possible roughness effects. The polishing was done using a Struers Tegramin-30. The blind longitudinal notches were machined by wire erosion, while the lateral notches were machined using a 30A15 disc in a Struers Accutom100 . 


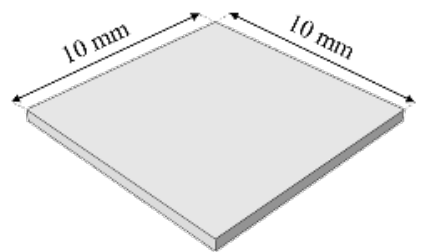

Standard

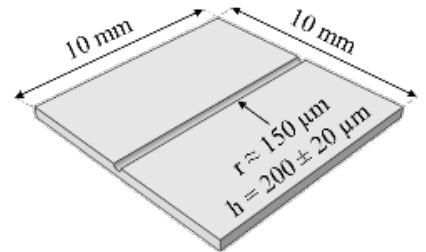

Blind longitudinal notch

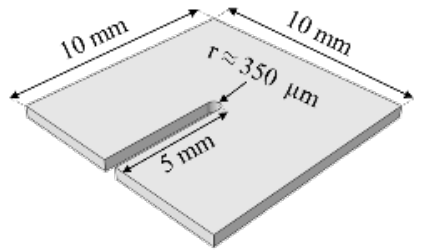

Lateral notch

Fig. 1. SPT specimen geometries

The SPT were done at room temperature at four different displacement rates: 5, 0,5, 0,05, and $0,005 \mathrm{~mm} / \mathrm{min}$. Each condition was tested both in air and in contact with the eGaIn. The measured force from the SPT was normalised by dividing it by the square of the thickness [16].

To have a good contact condition, the oxides present on the surface of the brass and the eGaIn were dissolved just before the test using a $1 \mathrm{M} \mathrm{HCl}$ solution $[13,17]$. For this, both metals were rinsed in the solution and the remaining acid was retired with cotton.

To observe the surface of the samples tested in contact with the eGaIn, these samples were cleaned just after the tests by immersing them in an ultrasonic bath with a $5 \%$ in vol. of $\mathrm{HNO}_{3}$ solution followed by a second immersion in a $1 \mathrm{M} \mathrm{NaOH}$ solution. Samples from all conditions were observed with the scanning electron microscope (SEM) Hitachi SU5000 to examine the cracking and the fracture surfaces.

For each condition (environment, geometry of the sample, strain rate), at least two tests were carried out. In the case of scattering, a third test or more were performed. Each SPT curve represents one of the tests performed in the mentioned condition.

\section{Results and discussion}

Using the standard SPT samples, we observed no signs of embrittlement on the brass tested in contact with the eGaIn. Indeed, the normalised force vs displacement curves are similar at any of the displacement rates tested, as shown in Figure 2.
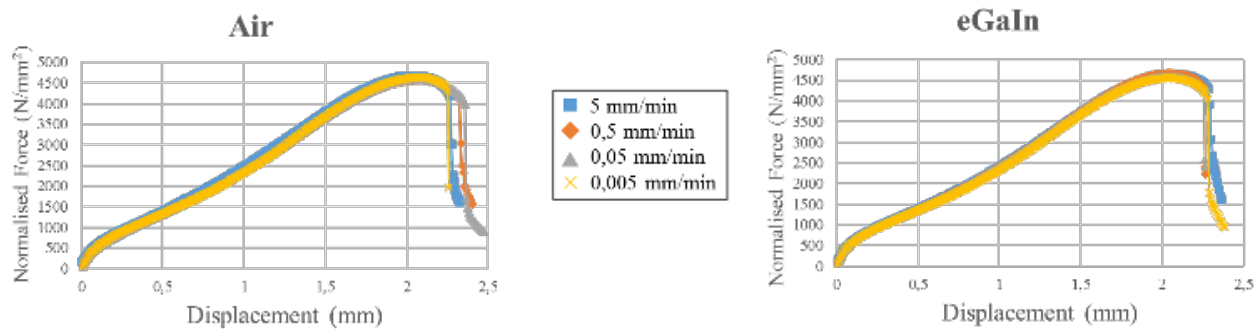

Fig. 2. SPT curves of flat samples in air and in contact with the eGaIn at different displacement rates

Furthermore, there is a similar fracture behaviour between the samples tested in air and those tested in contact with the liquid eGaIn, as observed in Figure 3. The cracks follow a circular pattern as expected on materials with a ductile fracture behaviour, which indicates that there was no LME. The secondary fractures, which propagate radially from the principal circular fracture, are greater and more numerous in the sample tested in contact with the eGaIn. However, in both samples, the secondary fractures have a ductile appearance. 

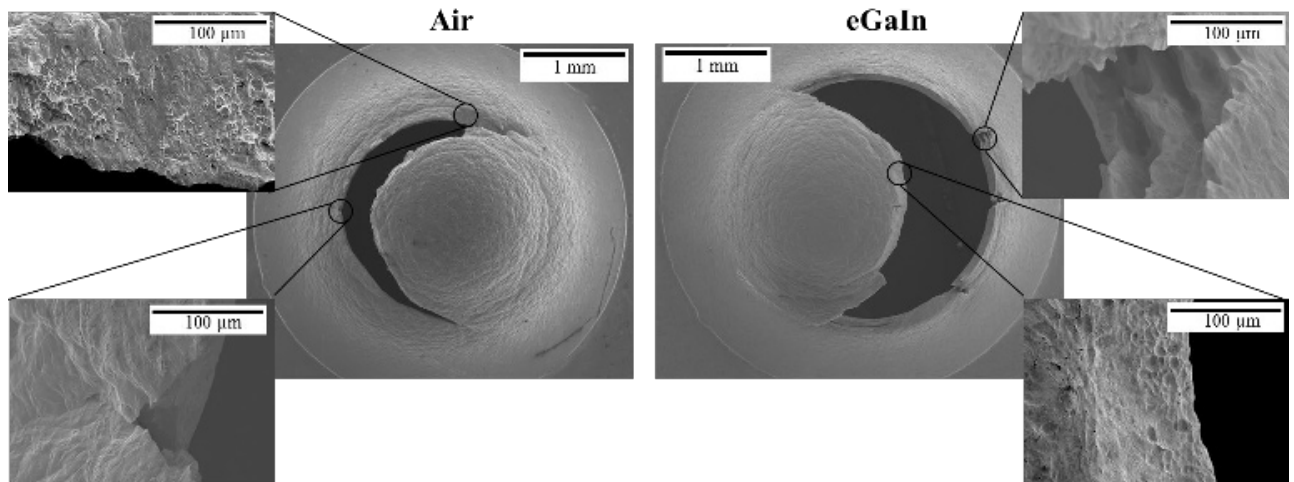

Fig. 3. Fractures of the SPT standard samples tested in air and in contact with the liquid eGaIn at a displacement rate of $5 \mathrm{~mm} / \mathrm{min}$

This indicates that the eGaIn does not affect the mechanical properties of the brass at these testing conditions. However, the occurrence of the LME is strongly dependent on the external conditions of the test, such as the strain rate and the stress $[2,4,5]$. In the previous tests, we cover a large range of strain rates but we did not detect LME. However, the use of different sample geometries changes significantly the stress state, which may facilitate the embrittlement of the solid.

For the samples with a blind longitudinal notch, Figure 4 shows the curves of two of them tested at $5 \mathrm{~mm} / \mathrm{min}$, one in air and the other in contact with the eGaIn. On one hand, the sample tested in air presents a sudden decrease in the force that is associated with the crack initiation, and then the decreasing of the force becomes smooth at some point of the crack propagation through the longitudinal notch. On the other hand, the sample tested in contact with the liquid eGaIn resembles the sample tested in air before the stage of crack propagation, but there is a second abrupt decrease in the force during crack propagation in this sample. This difference points to an effect of the eGaIn that affects exclusively the crack propagation and not the crack initiation.

\section{$5 \mathrm{~mm} / \mathrm{min}$}

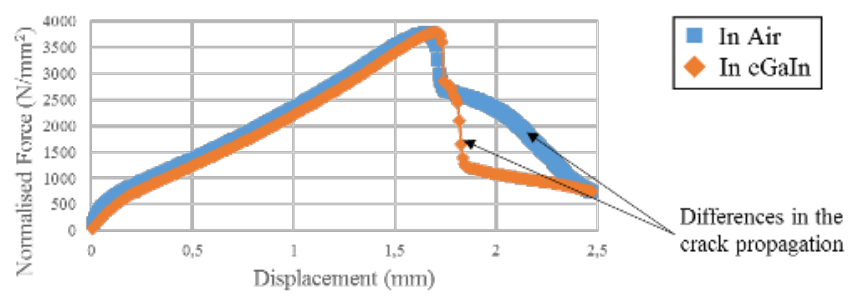

Fig. 4. SPT curves of samples with a blind longitudinal notch tested in air and in contact with the eGaIn at $5 \mathrm{~mm} / \mathrm{min}$

Furthermore, the SEM images in Figure 5 show the fractures of the SPT longitudinal notched samples tested at $5 \mathrm{~mm} / \mathrm{min}$. As expected, there are no significant differences in the centre of the fracture, which is the area of crack initiation. Moreover, in the extremes of the sample tested in air, there are dimples, which indicate a ductile fracture. This is also the case for the sample tested in contact with the eGaIn, but only on one of the fracture extremes (the upper extreme in Figure 5). However, the dimples in the sample tested in contact with the eGaIn are different since the cleaning operation (described in the previous section) degraded the sample surface. Nevertheless, there are signs of embrittlement by the eGaIn at the other 
end of the fracture (the lower extreme in Figure 5). This matches with the fact that there are only differences at the end of the normalised force vs displacement curves.
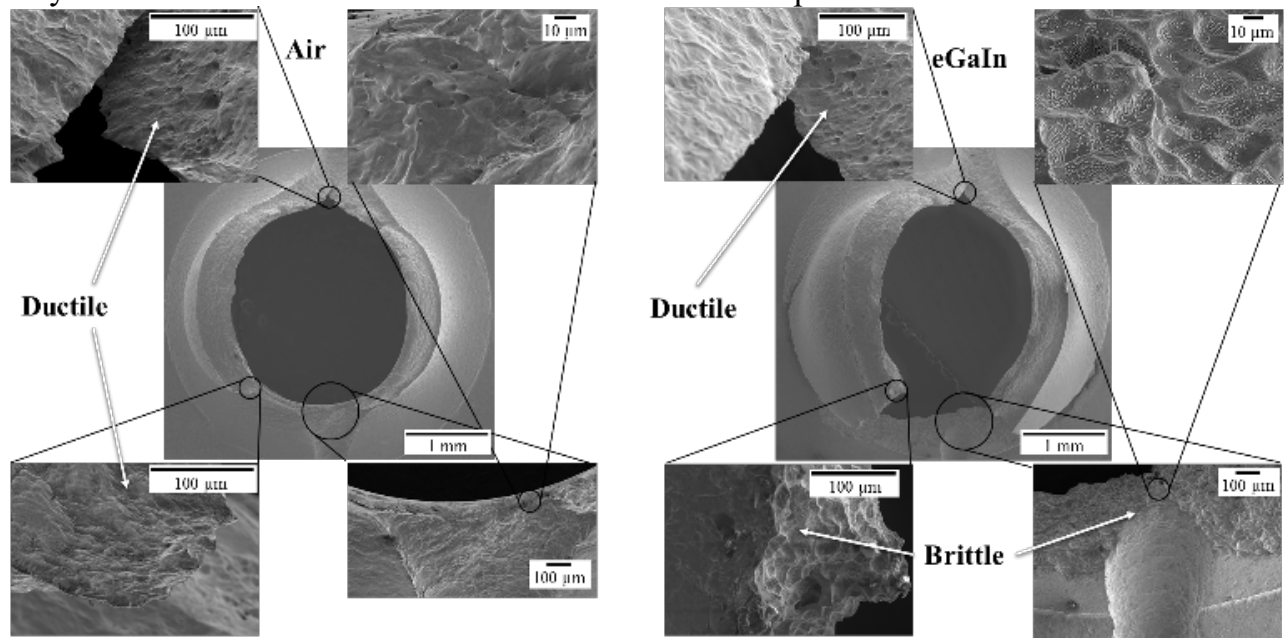

Fig. 5. Fractures of the SPT samples with a blind longitudinal notch tested in air and in contact with the eGaIn at a displacement rate of $5 \mathrm{~mm} / \mathrm{min}$

Even so, on the longitudinal notched samples tested at slower displacement rates $(0,5$ $\mathrm{mm} / \mathrm{min}$ ), we observe no differences between the sample tested in air and the sample tested in contact with the eGaIn. As depicted in Figure 6, all the samples showed no significant differences in their normalised force vs displacement curves, and the fracture behaviour is similar, with signs of ductility at the end of the fracture. Since all other test conditions remained unchanged, the lack of brittleness in these samples is related to the decrease in the strain rate.
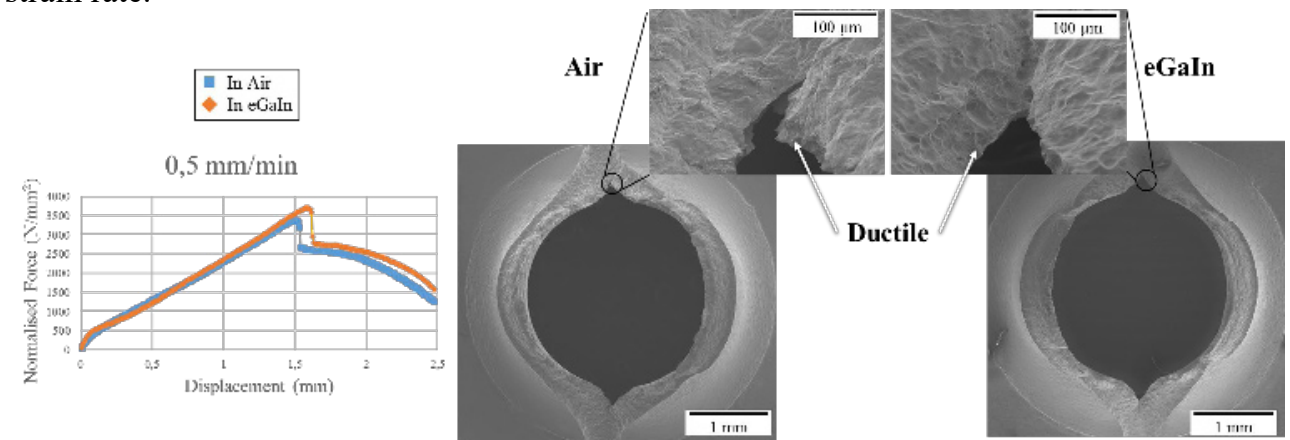

Fig. 6. Fractures of the SPT samples with a blind longitudinal notch tested in air and in contact with the eGaIn at a displacement rate of $0,5 \mathrm{~mm} / \mathrm{min}$

The samples with a lateral notch presented similar results as those with a blind longitudinal notch, as shown in Figure 7. In terms of the normalised force vs displacement curves, the point of initiation of the fracture is similar in air and in eGaIn, but there are differences during the propagation of the fracture, with that in liquid eGaIn being slightly more abrupt. However, the differences in the curves are less evident than for samples with a blind longitudinal notch. In terms of the fracture, the samples tested in air presented two cracks, both of which had dimples that indicate a ductile fracture. The samples tested in contact with the eGaIn only had one brittle fracture, which indicates LME. 

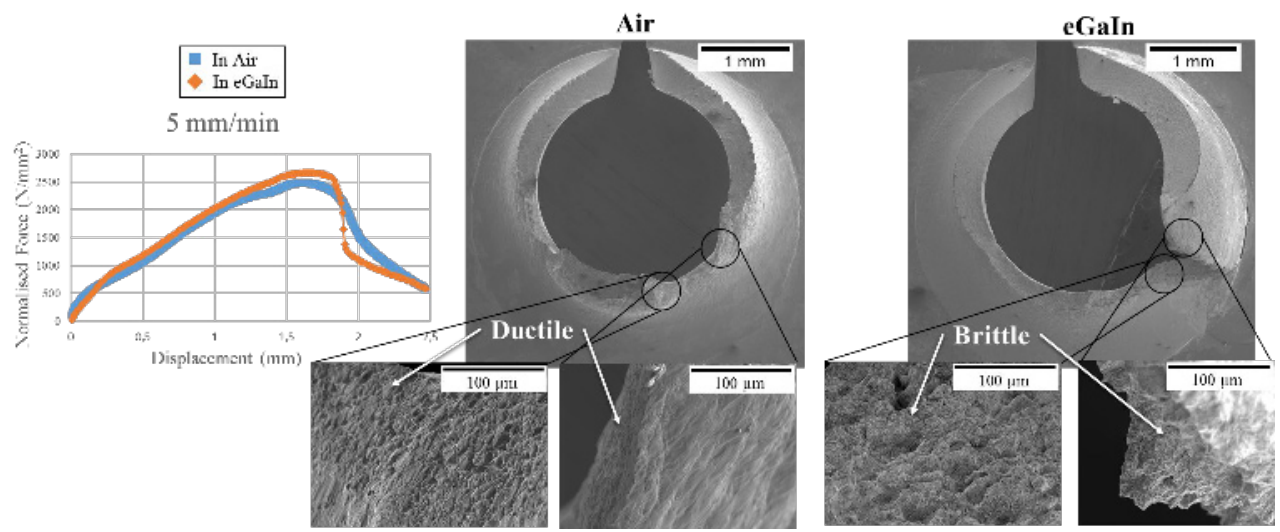

Fig. 7. Fractures of the SPT samples with a lateral notch tested in air and in contact with the liquid eGaIn at a displacement rate of $5 \mathrm{~mm} / \mathrm{min}$

The mechanical behaviour of the specimens with a lateral notch varies significantly from those with a longitudinal notch. The variations are linked to the differences in the stress distribution in the sample and the strain rate at the bottom of the notch. The fracture propagation is also different between geometries. For the longitudinal samples, the fracture must first propagate through the thickness of the sample and then through the longitudinal notch; while for the lateral notch, the crack initiates in thickness of the sample and does not have a delimited path to grow. These differences would explain why the impact of the eGaIn on the mechanical properties of the brass is more evident on the samples with a longitudinal notch, as all these mechanical conditions affect drastically the apparition of the LME.

Nevertheless, there was an effect of the eGaIn on the fracture behaviour of the brass $\mathrm{Cu}$ $30 \mathrm{wt} \% \mathrm{Zn}$ when using the notched specimens, but not when using the standard specimens. These results indicate that both notched geometries are more suitable to study the LME phenomenon than the standard flat geometries. However, we must note that in comparison with the standard geometries the notched samples need a more complex preparation. Especially for the longitudinal sample, as its fabrication can be made only with fine machining methods, such as wire electro erosion. Moreover, using a linear notch, the study of anisotropic effects would be more complex. Yet, the SPT on notches samples is a suitable test for little material availability.

The impact of the eGaIn on the fracture behaviour of the brass appears only during the crack propagation. This is related to the fact that in the SPT the stress state, the strain rate, and the magnitude of the plastic deformation applied to the solid are not constant through the sample. As consequence, the mechanical conditions and the degree of strain hardening are different at the point of crack initiation and during the crack propagation.

The fact that the effect of the eGaIn is only present during the crack propagation indicates that although there is LME of $\mathrm{Cu}-30 \mathrm{wt} \% \mathrm{Zn}$ by the liquid eGaIn its apparition is highly dependent on the stress-strain states and the hardening conditions, and it only occurs when the correct combination of these are present in the brass.

On one hand, the mechanical conditions before the crack initiation and during the first part of its propagation were not suitable for the start of the LME in this system. However, these conditions evolved along with the crack, and at some point of the crack propagation, the solid presented the necessary stress-strain state to enable the apparition of LME. On the other hand, as LME depends on the metallurgical state, the hardening of the sample enhanced the apparition of the embrittlement.

A numerical finite element analysis would allow calculating and comparing precisely the stress fields and the strain rate on the different specimens. 
Besides, the SPT showed that the brass $\mathrm{Cu}-30 \mathrm{wt} \% \mathrm{Zn}$ is more susceptible to the embrittlement by eGaIn at higher strain rates. This behaviour is similar to that observed on high-Mn steel in contact with liquid $\mathrm{Zn}$, which is a system that presents LME but that at slow strain rates a great fraction of solid intermetallic compounds forms and impedes the LME [18]. The same mechanism could affect the couple $\mathrm{Cu}-30 \mathrm{wt} \% \mathrm{Zn} / \mathrm{eGaIn}$ with the formation of the intermetallic $\mathrm{CuGa}_{2}$, which is stable at room temperature. In Ref. [19], the authors propose another mechanism of embrittlement at high strain rates; according to their work, variations in the strain rate would change the strain-hardening coefficient and hence the plastic deformation in the surface layers of the solid, facilitating the embrittlement at high strain rates. In contrast, in the T91 steel mechanically tested in contact with the lead-bismuth eutectic, there is a competition between the ductile and the brittle fractures, where higher strain rates promote a completely ductile fracture [20].

\section{Conclusions}

Using the Small Punch Test (SPT) with standard specimen geometries did not show any effect of the eGaIn on the mechanical properties of the $30 \mathrm{wt} \% \mathrm{Zn}$ brass at any of the displacement rates tested. The force vs displacement curves presented no differences when compared with those of the samples tested in air and the observations taken by scanning electron microscopy (SEM) showed a ductile fracture at all conditions.

By using the SPT on specimens with a blind longitudinal notch, we observed an effect of the eGaIn on the fracture behaviour of the brass. According to the curves and the analysed fractures, the eGaIn did not influence the initiation of the fracture but its propagation, changing it from ductile to brittle at a certain point of the fracture propagation. This indicates that the mechanical conditions for the LME appearance are only fulfilled during the fracture propagation.

The lateral notched specimens show similar results, with an effect of the eGaIn in the propagation of the fracture, as shown by the SEM images. However, the differences are less evident in the force vs displacement curves.

Indeed, notched SPT specimens are more appropriate to screen the system $\mathrm{Cu}-30 \mathrm{wt} \%$ $\mathrm{Zn} / \mathrm{eGaIn}$ for LME. The specimens with a blind longitudinal notch are more suitable for this purpose than those with a lateral notch. However, the preparation of these specimens requires fine machining tools.

The embrittlement effect in the notched specimens is present only at the higher displacement rate, indicating that the $30 \mathrm{wt} \% \mathrm{Zn}$ brass is more susceptible to LME by the eGaIn at higher strain rates.

This work was done at the Unité Matériaux et Transformations (UMET) with the support of the Agence nationale de la recherche (ANR) under the project ANR GauguIn ( ${ }^{\circ}$ ANR-18-CE08-0009-01).

Acknowledgements to the valuable technical support of D. Creton, J. Golek, and A. Fadel.

Observations and analyses by SEM were performed at the electron microscope facility at the University of Lille with the support of the Chevreul Institute, the European FEDER, and the Région Hauts-de-France (France).

\section{References}

1. M. G. Nicholas and C. F. Old, "Review Liquid metal embrittlement," J. Mater. Sci., vol. 14, pp. 1-18, (1979).

2. B. Joseph, M. Picat, and F. Barbier, "Liquid metal embrittlement: A state-of-theart appraisal,” EPJ Appl. Phys., vol. 5, no. 1, pp. 19-31, (1999).

3. D. G. Kolman, "A review of recent advances in the understanding of liquid metal 
embrittlement," Corrosion, vol. 75, no. 1, pp. 42-57, (2019).

4. M. M. Shea and N. S. Stoloff, "Embrittlement of beta-brass alloys by liquid metals and aqueous ammonia," Mater. Sci. Eng., vol. 12, no. 5-6, pp. 245-253, (1973).

5. P. J. L. Fernandes and D. R. H. Jones, "The effects of microstructure on crack initiation in liquid-metal environments," Eng. Fail. Anal., vol. 4, no. 3, pp. 195204, (1997).

6. I. Serre and J. B. Vogt, "Liquid metal embrittlement of T91 martensitic steel evidenced by small punch test," Nucl. Eng. Des., vol. 237, no. 7, pp. 677-685, (2007).

7. R. Lacalle, J. A. Álvarez, and F. Gutiérrez-Solana, "Use of Small Punch Notched Specimens in the Determination of Fracture Toughness," in Volume 6: Materials and Fabrication, Parts A and B, (2008), pp. 1363-1369.

8. E. Martínez-Pañeda, T. E. García, and C. Rodríguez, "Fracture toughness characterization through notched small punch test specimens," Mater. Sci. Eng. A, vol. 657, pp. 422-430, Mar. (2016).

9. A. Legris, G. Nicaise, J. B. Vogt, J. Foct, D. Gorse, D. Vançon, "Embrittlement of a martensitic steel by liquid lead," Scr Mater, vol. 43, no. 11, pp. 997-1001, (2000).

10. A. Hojná, F. Di Gabriele, J. Klecka, and J. Burda, "Behaviour of the steel T91 under uniaxial and multiaxial slow loading in contact with liquid lead," J. Nucl. Mater., vol. 466, pp. 292-301, (2015).

11. D. E. Tyler and W. T. Black, "Introduction to Copper and Copper Alloys," in Properties and Selection: Nonferrous Alloys and Special-Purpose Materials, ASM International, (1990), pp. 216-240.

12. S. Liu, K. Sweatman, S. McDonald, and K. Nogita, "Ga-based alloys in microelectronic interconnects: A review," Materials (Basel)., vol. 11, no. 8, pp. 120, (2018).

13. Q. Xu, N. Oudalov, Q. Guo, H. M. Jaeger, and E. Brown, "Effect of oxidation on the mechanical properties of liquid gallium and eutectic gallium-indium," Phys. Fluids, vol. 24, no. 6, p. 063101, Jun. (2012).

14. H. Nichols and W. Rostoker, "Ductile-brittle transition in alpha brass," Acta Metall., vol. 8, no. 12, pp. 848-850, (1960).

15. M. Bruchhausen et al., "European standard on small punch testing of metallic materials,” Ubiquity Proc., vol. 1, no. S1, p. 11, Sep. (2018).

16. T. E. García, C. Rodríguez, F. J. Belzunce, and C. Suárez, "Estimation of the mechanical properties of metallic materials by means of the small punch test," J. Alloys Compd., vol. 582, pp. 708-717, Jan. (2014).

17. D. Kim et al., "Recovery of nonwetting characteristics by surface modification of gallium-based liquid metal droplets using hydrochloric acid vapor," ACS Appl. Mater. Interfaces, vol. 5, no. 1, pp. 179-185, (2013).

18. J.-H. Kang, S.-H. Hong, J. Kim, and S.-J. Kim, "Zn-induced liquid metal embrittlement of galvanized high-Mn steel: Strain-rate dependency,” Mater. Sci. Eng. A, vol. 793, p. 139996, Aug. (2020).

19. V. V. Popovich and I. G. Dmukhovskaya, "Rebinder effect in the fracture of Armco iron in liquid metals," Sov. Mater. Sci., vol. 14, no. 4, pp. 365-370, (1979).

20. Z. Hamouche-Hadjem, T. Auger, I. Guillot, and D. Gorse, "Susceptibility to LME of 316L and T91 steels by LBE: Effect of strain rate," J. Nucl. Mater., vol. 376, no. 3, pp. 317-321, Jun. (2008). 